\title{
Tree Based Localization Model for Line Following Robots
}

\author{
Tanvir Ahmed ${ }^{1}$, Khandakar Mahbubul Islam ${ }^{2}$, Md. Faiz Ahmed ${ }^{3}$, Rakib Hossain ${ }^{4}$ \\ and Md. Mahbubur Rahman ${ }^{5}$ \\ Department of Computer Science \& Engineering \\ Bangladesh University of Business \& Technology (BUBT) \\ Dhaka, Bangladesh \\ tanvircse10@gmail.com ${ }^{1}$,khmahbub20@gmail.com ${ }^{2}$, \\ shipuahmed01@gmail.com ${ }^{3}$, agilerakib7728@gmail.com ${ }^{4}$, \\ mahabub.cse.buet@gmail.com ${ }^{5}$
}

\begin{abstract}
Today automated frameworks are broadly utilized as a part of the business specifically for assignments, for example, welding, painting and bundling. All of these automated systems are in the form of manipulators that carry out repetitive motion. For large scale transportation such robotics systems are not particularly practical and therefore automatic transportation systems have been developed either as numerically controlled systems like automatic warehouses or through use of automatic guided vehicles (AGVs). Almost all AGVs use a guide-path system where they track a buried Wire on the floor or utilize other forms of artificial landmarks. In this paper we design an algorithm for a line follower based service robot which could be applicable to various practical automation systems. Here, a new localization algorithm has been proposed to localize the mobile robot in an area. Various experiments show that the proposed algorithm could accurately evaluate the localization performance. It can be called using a web application. A SPI based wireless system has been used to send user command to robot and get data from robot. It has a sonar device through which it can detect obstacles and hold that position for a while. By using the gear motors the robot can able to move faster via following the line. It's extremely basic and simple to utilize. This gadget is practical, productive in utilizing force and easy to understand enough to make our day by day works less demanding.
\end{abstract}

Keywords: Arduino, AGVs, SPI, RFID, Localization, LRF, IR sensor, Sonar sensor

\section{Introduction}

Robotics is one of the quickest developing zones of research. It will contribute much to the future and has many promising properties. Nowadays, robotics is everywhere and is affecting our lives tremendously. Starting from computers and ending with space crafts. Humanity is getting more dependent on robots and every year there are thousands of researches and projects that add new contributions to science and engineering of robotics [1].

New algorithms that improve old techniques as well as new designs and new robots are presented continuously. The ultimate goal is to develop a fully automated machine with all its required sensors to sense its surroundings and make behavioral decision independently.

Additionally, the machine that would work in a social environment should have also a reasonable size and behaviors based on perceived personality. Autonomous behavior is 
one of the most important aspects related to modern robotics. Localization and navigation are part of automatic behavior and they are one of the most quickly developing fields of robotics research presently [12].

\subsection{Formal Definition}

We can define robots as follows:

A: a machine that looks like a human being and performs various complex acts (as walking or talking) of a human being; also : a similar but fictional machine whose lack of capacity for human emotions is often emphasized.

$B$ : an efficient insensitive person who functions automatically is known as robot.

Basically robots are some kind of device that automatically performs complicated often repetitive tasks and they have a mechanism guided by automatic controls [12].

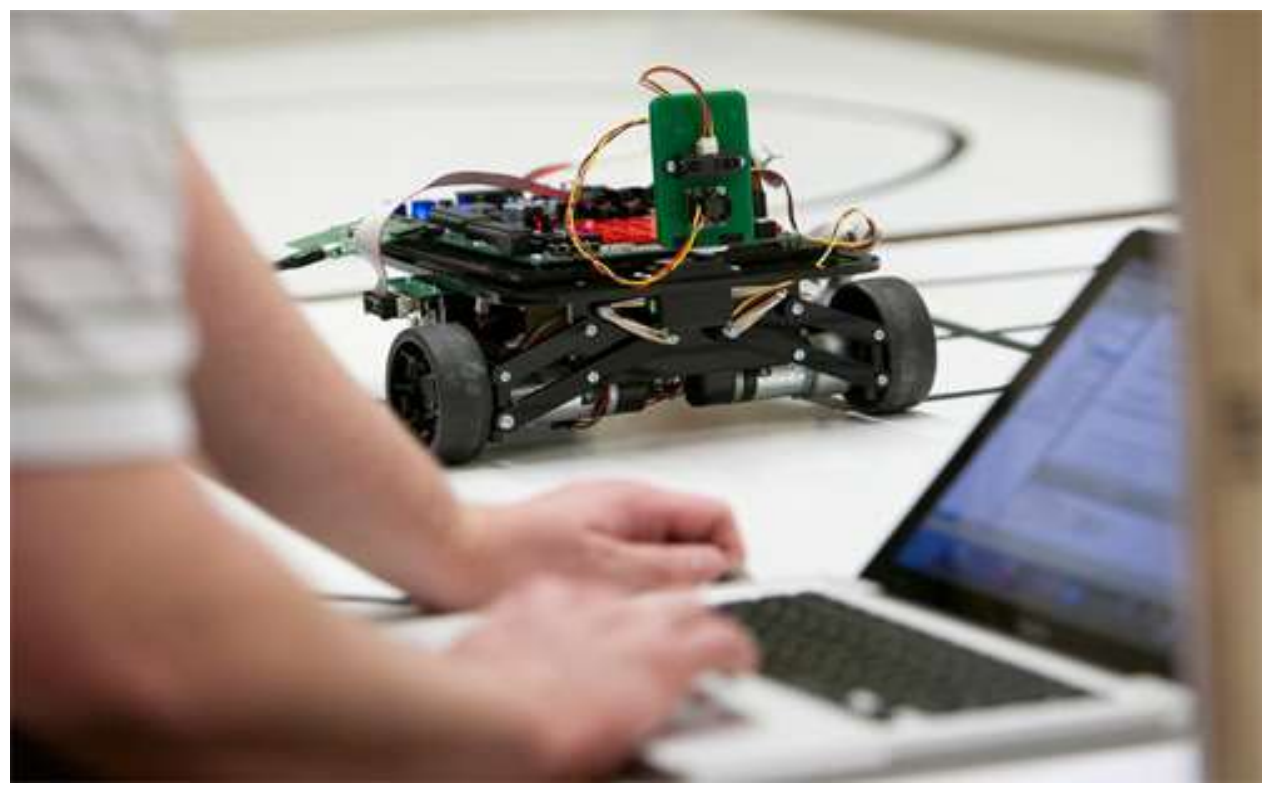

Figure 1. Robotics

\subsection{Types of Robot}

These days, robots do various assignments in numerous fields and the quantity of employments depended to robots is becoming consistently. That is the reason as I would like to think one of the most ideal routes how to partition robots into sorts is a division by their application. As follows:

Industrial robots - Industrial robots will be robots utilized as a part of a modern assembling environment. Generally these are enunciated arms particularly produced for such applications as welding, material taking care of, painting and others. In the event that we judge absolutely by application this sort could likewise incorporate some mechanized guided vehicles and different robots.

Domestic or household robots - Robots utilized at home. This sort of robots incorporates numerous entirely extraordinary gadgets, for example, mechanical vacuum cleaners, automated pool cleaners, sweepers, canal cleaners and different robots that can do distinctive tasks. Additionally, some reconnaissance and telepresence robots could be viewed as family unit robots if utilized as a part of that environment.

Medical robots - Robots utilized as a part of prescription and therapeutic foundations. As a matter of first importance surgery robots. Additionally, some mechanized guided vehicles and perhaps lifting helpers. 
Service robots - Robots that don't fall into different sorts by use. These could be diverse information gathering robots, robots made to flaunt innovations, robots utilized for research, and so on.

Military robots - Robots utilized as a part of military. This kind of robots incorporates bomb transfer robots, distinctive transportation robots, observation rambles. Regularly robots at first made for military purposes can be utilized as a part of law requirement, pursuit and safeguard and other related fields.

Entertainment robots - These are robots utilized for amusement. This is an exceptionally general classification. It begins with toy robots, for example, robosapien or the running wake up timer and finishes with genuine heavyweights, for example, explained robot arms utilized as movement test systems.

Space robots - I'd jump at the chance to single out robots utilized as a part of space as a different sort. This sort would incorporate robots utilized on the International Space Station, Canadarm that was utilized as a part of Shuttles, and additionally Mars meanderers and different robots utilized as a part of space.

Hobby and competition robots - Robots that you make. Line adherents, sumo-bots, robots made only for entertainment only and robots made for rivalry.

\subsection{Robot Localization}

Localization is a process that occurs inside the robot "brain" to locate the robot in a previously known map. In other words, the map is already located in the robot memory and is based on landmarks of the environment that the robot perceives. Robot uses sonar, camera, Laser Range Finder (LRF) and stereo vision devices like Kinect to perceive the environment. Using localization and sensors, robot will be able to detect its position like $\mathrm{x}, \mathrm{y}$ coordinates as well as its orientation in the map. Even the sensing for localization process is not as easy as presented above because there are many types of sensors and a sensor fusion are required in the process. As long as there are sensors, there are noise and errors which cause uncertainty about all data produced by sensors. Always, without a refining algorithm, the outcome of the process of locating the robot position will diverge. In this case, if we need our robot to be in the hall room, after a while it will find itself in the kitchen! Nevertheless, filters are used by the robot in order to figure out the best estimation of its location and position. Methods of using a filter to refine signals coming from the sensors in order to determine the robot's position will be the main topic of this thesis $[2,13]$.

The issue of robot confinement comprises of answering the question Where am I? From a robot's point of view. This means the robot has to stand out its location relative to the environment. When we talk about location, pose, or position we mean then $\mathrm{x}$ and $\mathrm{y}$ coordinates and heading direction of a robot in a global coordinate system. The localization problem is a critical issue. It is a key part in numerous successful selfsufficient robot frameworks. On the off chance that a robot does not know where it is with respect to the earth, it is hard to choose what to do [3].

The robot will in all probability need at any rate some thought of where it is to have the capacity to work and act effectively. By a few creators the robot confinement issue has been expressed as the most basic issue to giving robots genuinely independent capacities [13]. 


\subsection{Robot Navigation}

Navigation is the next step taken by the robot when it knows its position. Localization is the main part here as the robot must know its position precisely while driving from point to point [4]. The navigation scenario will be:

1. The robot knows its essential position.

2. Robot receives an order to go to a different position (or set this goal by himself)

3. Robot creates a trajectory step by step from its start point to its destination point. This trajectory is first specified as a path in the map and then it is converted to control commands for the robot.

4. Starts moving.

5. Keep localizing itself while moving as it has to calibrate itself every while.

\section{Our Inspiration}

Intelligent devices are becoming common in our daily life day by day. New ideas are updating and new technologies are implementing to make these devices more robust and cheap. That's why the idea of using robots for daily works becoming popular.

Arduino is an open-source microcontroller in view of simple to-utilize equipment and programming. Interfacing different gadgets with Arduino circuit sheets are making the new developments for the robotics enterprises. In robotics, IR sensor is utilized for identifying the shading objects, in spite of the fact that it works by utilizing a particular light sensor to recognize a select light wavelength in the Infra-Red range. Ultrasonic or sonar sensor is utilized for distinguishing the range amongst indoor and outside articles. Ultrasonic or sonar sensors produce high recurrence sound waves and gauge the resound which is recognized back by the sensor, measuring the time interim between sending the flag and accepting the reverberate to decide the separation to a question [1].

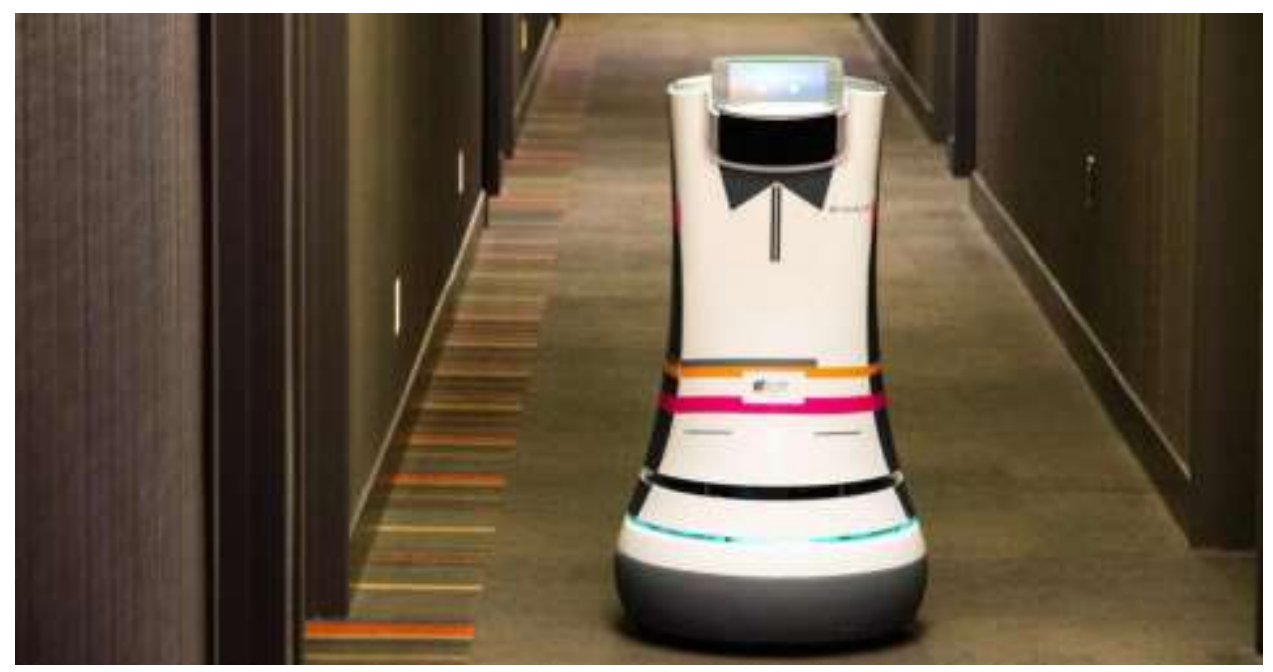

Figure 2. SaviOne (Hotel Service Robot)

In our paper, we have discussed about a path finder robot with the obstacle detections, the robot can be used for home or business applications. We have inspired about this project from the line follower robot competitions and hotel service robot "SaviOne" built by the company named "Savioke". Now we can see these robots working in Starwood chain hotel Aloft at Cupertino in California. They have named this robot as A.L.O [9]. 


\section{Thesis Outline}

\subsection{Background Analysis}

These days heaps of businesses utilizing numerous sorts of robots and every robot have its own particular purposes. A few robots can move one place to another which takes after a way. Toward the starting running a robot with an obscure way was excessively troublesome then analysts have presented with a name way discoverer. A way discoverer robot is essentially a robot intended to take after a line or way which is as of now predefined and foreordained by the client. The way is as straightforward as a white or dark hued line on the floor [8].

There are numerous explores about the way discovering robots one of these looks into additionally said about way discovering robot is utilized as server administration; thought of learning and this thought is utilized to explore through an impediment freeway from a beginning position to a known objective position on the obscure environment.

Another way discoverer robot is additionally composed with the RFID based application which comes from no express prerequisites and goes for client gathering to achieve the objective inside a no well-known or absolutely obscure grounds range without taking any assistance outside.

For a portion of the way discoverer utilized multi sensors to satisfy its principle targets. In our venture we have executed multi sensors for conferring different works. For finding the way and tailing it we have utilized the IR sensor by means of which distinguishes the dark line on white surface with high differentiated shading. The one of the essential sensor that we utilized is impediments sensor for the predefined and foreordained way [10].

Essentially while taking after a way confront numerous sorts of impediments; to stay away from or move these we require either picture preparing which can recognize the articles through pictures or sonic sensors which can distinguish the range between the items and the robot itself.

As per the insights it demonstrates that these days numerous robots are utilizing for enterprises as well as helpful for the independent venture like eatery, workplaces, schools, airplane terminal and so on spots and family unit purposes. For the family unit robots uses are step by step expanding and before the end of the 2016 it will cross more than 15000 units.

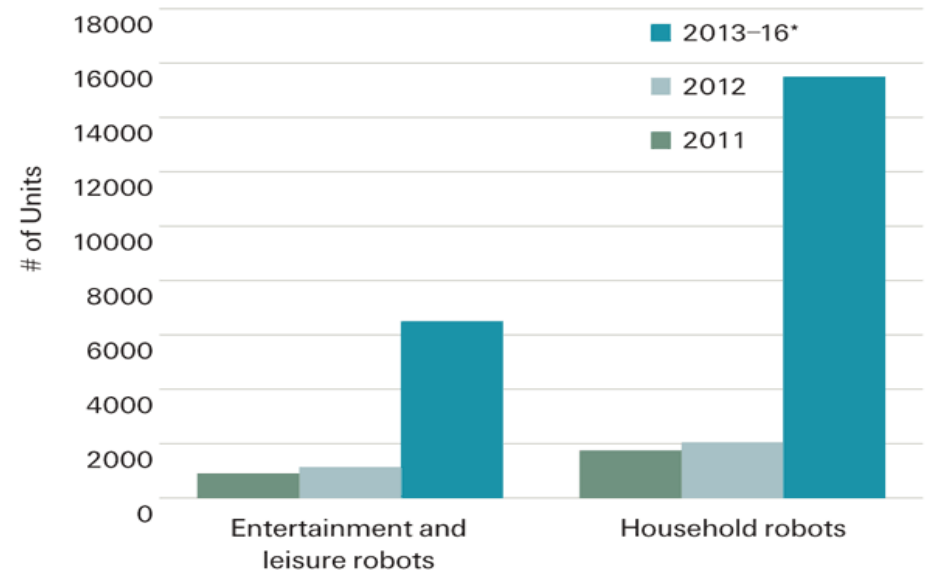

Figure 3. Statistics of Household Robots

Well, this is our proposed model. It is very much similar to conventional model, but the difference is in input seed $\&$ the arithmetic formula of generator. 


\subsection{Research Methodology}

A large portion of enterprises are utilizing robots for some reasons way discoverer is one of them. Particularly auto ventures and tech businesses are utilizing the way discovering robot to finish the mass measure of works. As of late family unit robots are being worked to serve as business or home applications as like server administration. Numerous autos are working with the way discovering thoughts which can act naturally drivable as like Google auto, Audi auto and Tesla auto, the possibility that result finding the way and take after the way until it achieves its own objective.

These days path finding robot is utilizing as a part of Mars operations as to study the earth on Mars. A few models have been presented in light of a change of strategies alongside some limitation with each.

This research is fundamentally gone for actualizing the home or business applications robots that is fit to serve any known territories. It can recognize obstacles to protests all alone way. The entire theory is finished by utilizing the Arduino IDE where way discoverer and impediments recognition is done and web application is worked to call the robot close to a client area. To confirm the culmination of the venture we have tried the sensors independently and consolidated with body for a few times to assess and idealizing the more exactness we needed to change the body structure for a few times.

\subsection{Implementation}

The whole work is divided in to 3 major parts:

1. Mechanical part

2. Electrical part

3. Software part

On mechanical part we have deigned the body along Gear motors, sonar sensor and IR sensor array.

On electrical part we have implemented the Arduino Uno along with the nRF24L01 RF Board device and Mega along with the L293D Motor Controller and the nRF24L01 RF Board device including the sensors.

Table 1. Body Parts

\begin{tabular}{|c|c|}
\hline Name of Part & Item Setup \\
\hline Mechanical part & Body \\
\cline { 2 - 2 } & Gear Motor \\
\cline { 2 - 2 } & Sonar Sensor \\
\cline { 2 - 2 } & IR Sensor Array \\
\hline Electrical part & Arduino Uno \\
\cline { 2 - 2 } & Arduino Mega \\
\cline { 2 - 2 } & L293D Motor Controller \\
\cline { 2 - 2 } & nRF24L01 \\
\cline { 2 - 2 } & LM2596 Step Down Module \\
\hline \multirow{5}{*}{ Software part } & Arduino IDE \\
\cline { 2 - 2 } & NetBeans IDE \\
\cline { 2 - 2 } & XAMPP(Apache/Mysql) \\
\cline { 2 - 2 } & User Interface(Web based application) \\
\hline
\end{tabular}

At the end on software part we have designed the algorithms by using the Arduino IDE, web application for the user interfaces so user can give command easily and a Java program which relays user commend to the robot. The Java program \& the web application shares a common MySQL database. The system that we designed to handle 2 different works:

1. Path finder design 
2. Robot monitoring

\section{Robot End:}

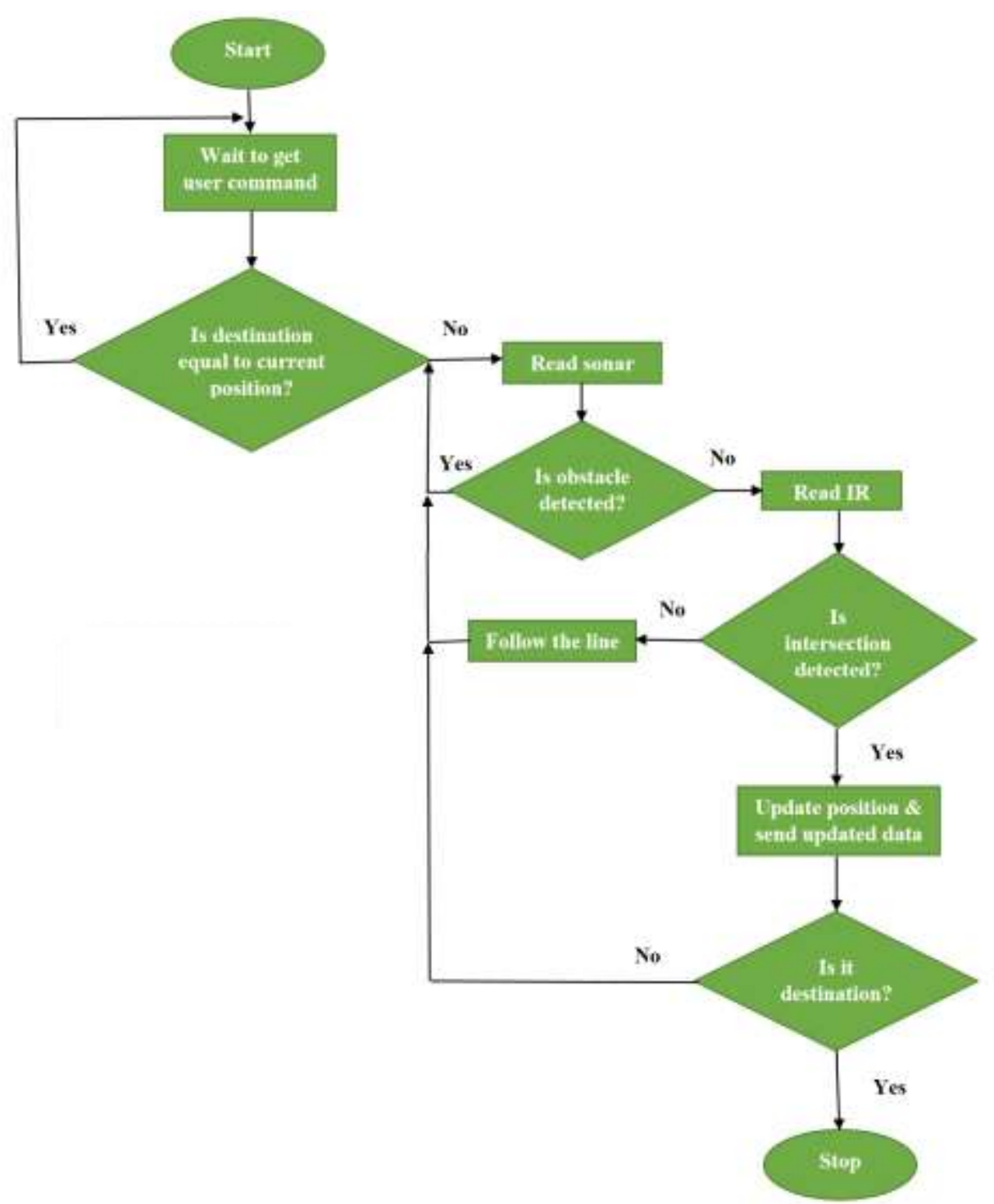

Figure 4. Mechanism of Robot End

\section{User End:}




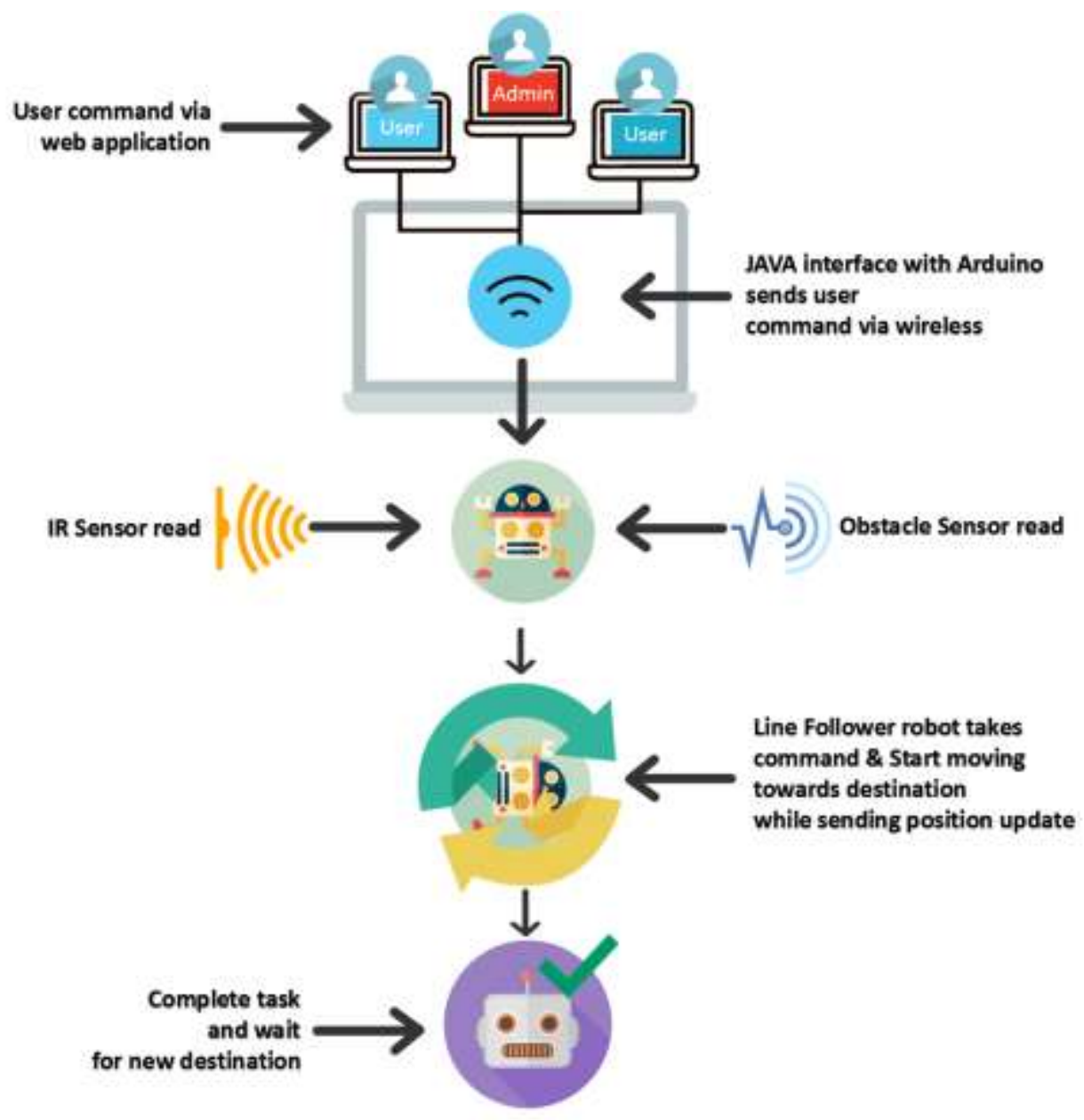

Figure 5. Mechanism of User End

Both user end and robot end are connected via the Java application with nRF24L01 connection. By default when a user make a call from a location the Java application forwards the command to the robot if it is idle. After connecting the robot will reach the location by following its path; the robot will start to move forward, left, right or turn 180 degree by reading the IR sensor and considering the destination. On the path if there are any obstacles detected by sonar sensor the bin will stop and wait until the obstacle object moved from the path. As there are defined location, the robot will stop from which location are being called by the user, rest of the locations will be ignored. After few seconds the robot will start to move; if the robot gets another call from another user it will respond to user as the same thing. After completing the task the robot will rest until it gets any call.

\section{Path Finder Control Design}

Implementing the prototype is one of the biggest challenges in our project. There are many reasons why it is not easy to implement one of them is perfecting the structure, which helps the rest of the parts to hold. The second is to write the code and choose the appropriate pin for the sensors. We have divided the implementation on two parts first is Mega second is Uno [5]. 


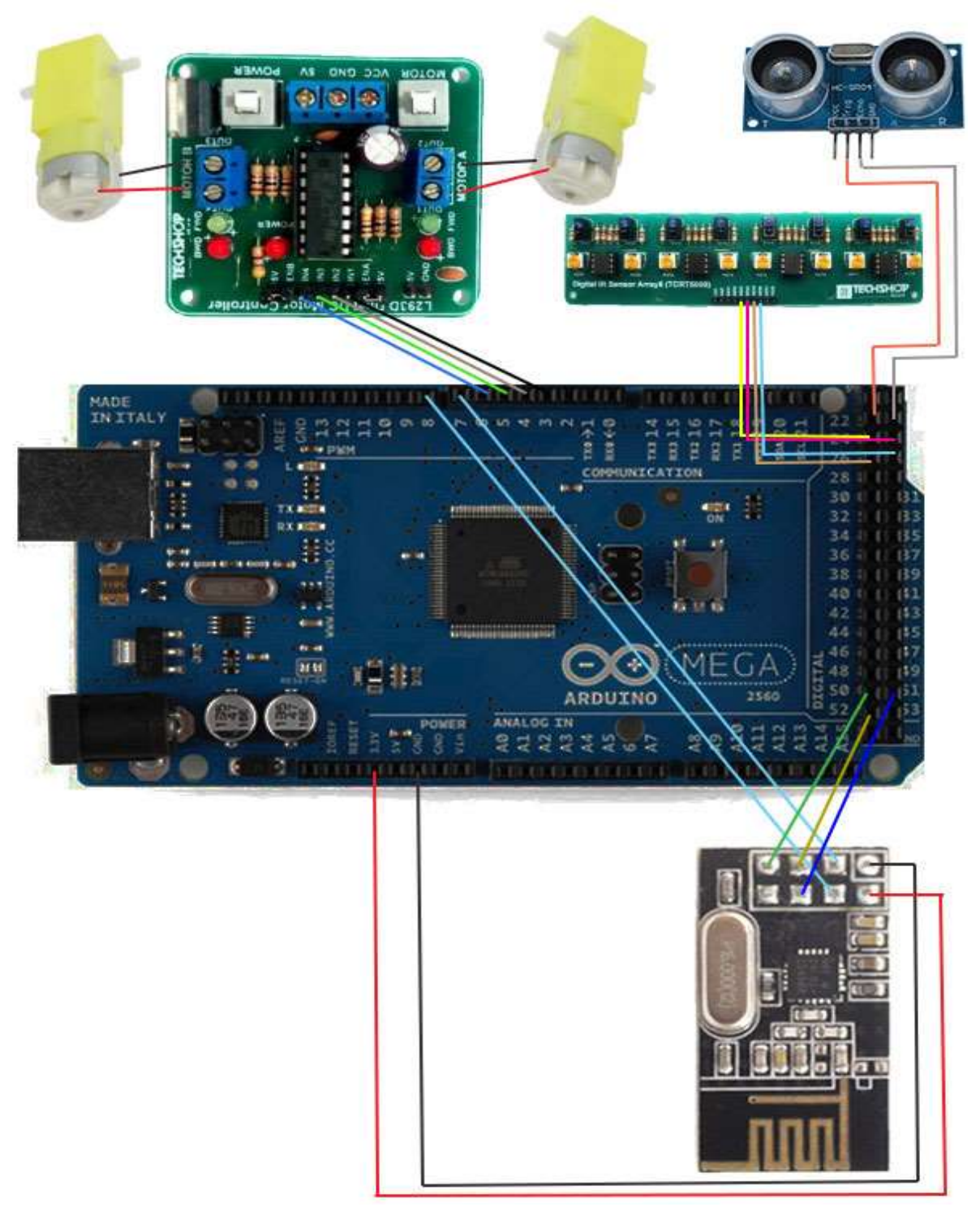

Figure 6. Path Finder Control

On the first part we have used Arduino Mega along with the sonar sensor, IR sensor and motor controller L293D. IR sensor take the analog reading of black and white color for the path following and motor controller is connected with two motors which controls the motors movements according to sensing of the path. If more than two IR sensor detects black line colored position, it is selected as an intersection point. On the other hand if sonar sensor is used for detecting the obstacles and the distance between the obstacles and the moving objects. HC-SR04 ultrasonic sensor uses sonar to assume distance to an object like bats or dolphins do. It offers excellent non-contact range detection with high accuracy and stable readings in an easy-to-use package from $2 \mathrm{~cm}$ to $400 \mathrm{~cm}$ or 1 " to 13 feet. If sonar sensor detects any obstacles within its ranges two motors will be stop until the obstacle is moved. An nRF24L01 RF board device is connected, so that user can call the robot to user's location via web application. The most important benefits of using SPI based communication is easy to connect and can work on limited areas. 


\section{Base Station Design}

On this part, we have connected an nRF24L01 RF board with Arduino Uno. A bread board is used to connect the Arduino Uno's $3.3 \mathrm{~V}$ pin with a $10 \mu \mathrm{F}$ capacitor to power the nRF24L01 RF board. After connecting all the devices the Arduino Uno is connected with a computer using an USB cable. Then we run the Java application. The mechanism is designed like this when user puts a command using the web application the command is stored in a MySQL database. The Java application fetches the command from the database and sends the data to the robot when the robot is idle [6].

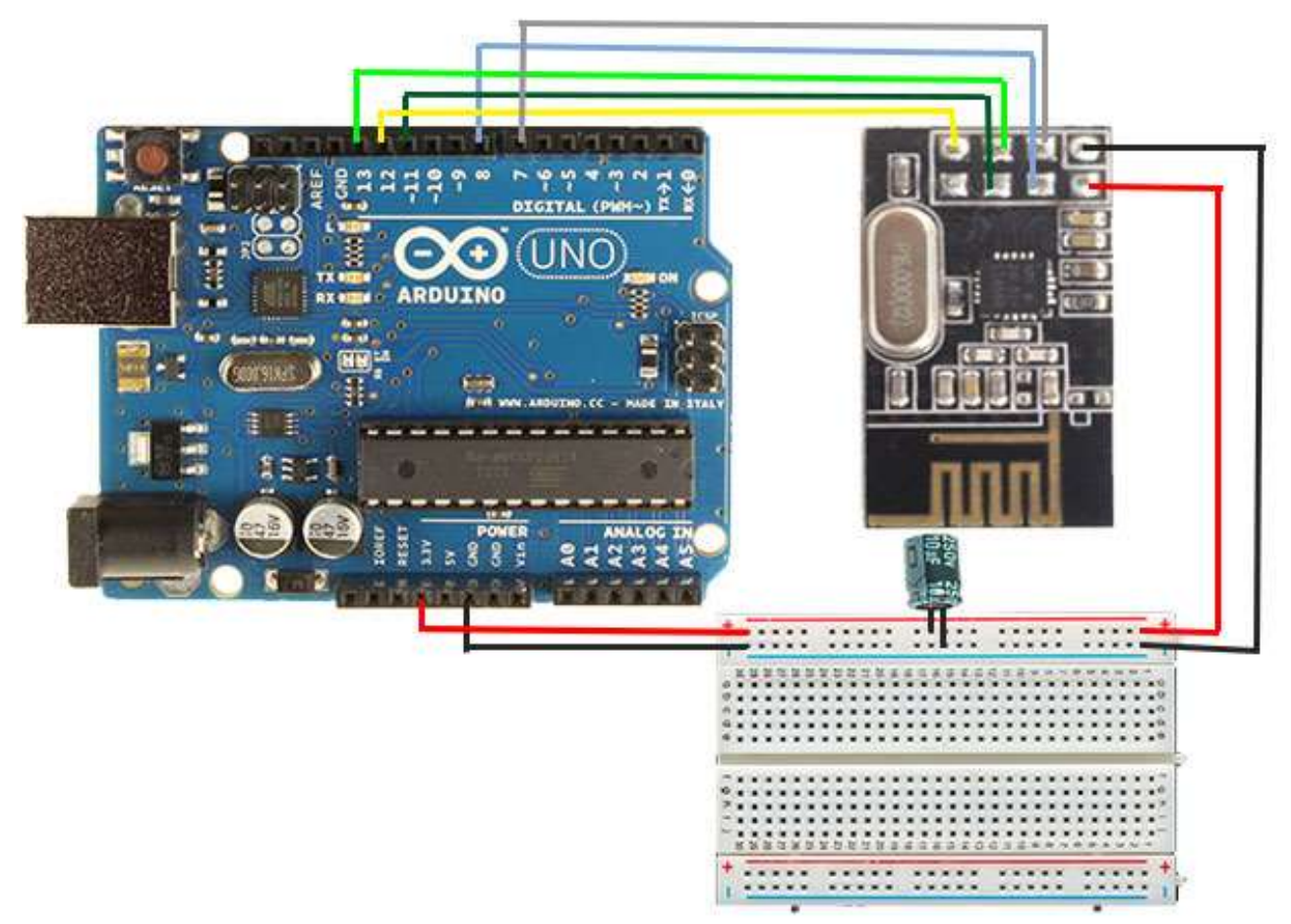

Figure 7. Base Station

When the robot send its updated position after reaching an intersection point; the Java application stores the data in a MySQL database. The web application uses the data to show the robot's position in the map. Users can see the robots position from the web application's map page any time.

\subsection{Simulation \& Analysis}

Our main objective of this research is to provide an intelligent algorithm for line following robots. That's why we need to apply a physical survey and observe our algorithm through a real robot simulation. In this connection here we use Arduino.

Our robot consist of an acrylic base with two gear motors, two compatible wheels, a ball caster, and other accessories. We have used a Vero board for the $1^{\text {st }}$ floor base of our robot. There are many classifications of arduino. It is a microcontroller. The Arduino board is the brain of the robot, as it will be running the software that will control all the other parts [7]. The programming is done in this part.

Chassis Specification:

1. Dimensions: 7.72 in $x 4.13$ in $x 0.12$ in $(19.6 \mathrm{~cm} \times 10.5 \mathrm{~cm} \times 0.3 \mathrm{~cm})$

2. Weight: $14.29 \mathrm{oz}(405 \mathrm{~g})$

Vero Board Specification:

1. Dimensions: $20.5 \mathrm{~cm} \mathrm{x} 9.5 \mathrm{~cm}$ 


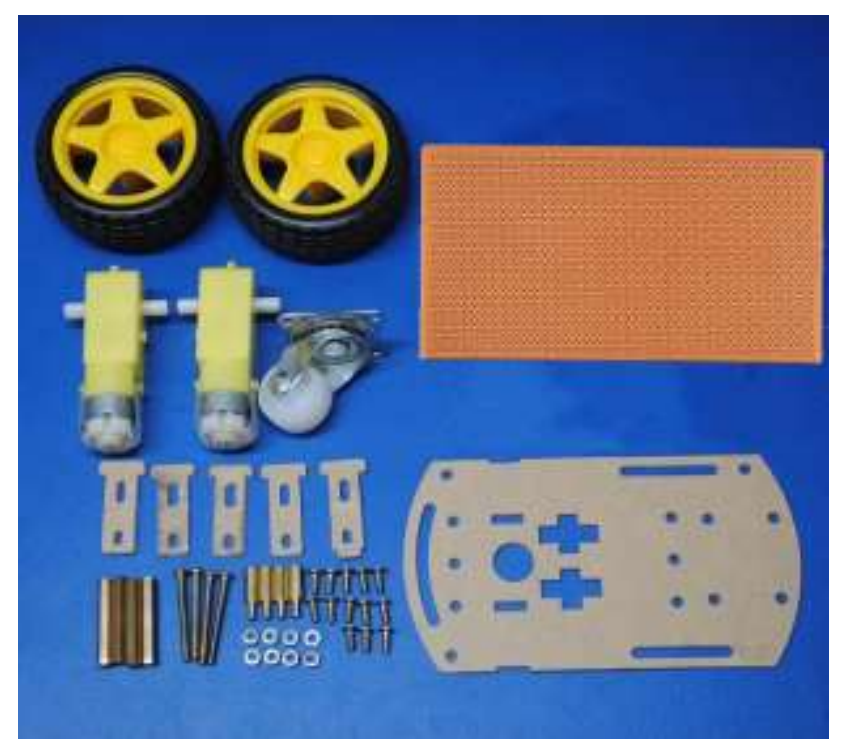

Figure 8. Accessories to Assemble

\section{Prototype 1}

We defined thesis scope and topic. We revised the topic, studied about its benefit and work horizon and then we selected one from many. Three of the group members started the research work. Meeting with the group member had been done on regular basis. We discussed and made the working plans and basic diagrams. Based on the blueprint we have done our first poster presentation and submitted a report including each and every detail. We have designed the 1st prototype with less efficiency. The prototype was made with two gear motors, one free wheel and IR sensor array. The idea that came across of our mind with the $1^{\text {st }}$ prototype is to provide service at restaurants, ground floor of hotels, office floors and university building areas types of places. Our best concern was to make the prototype good on line following.

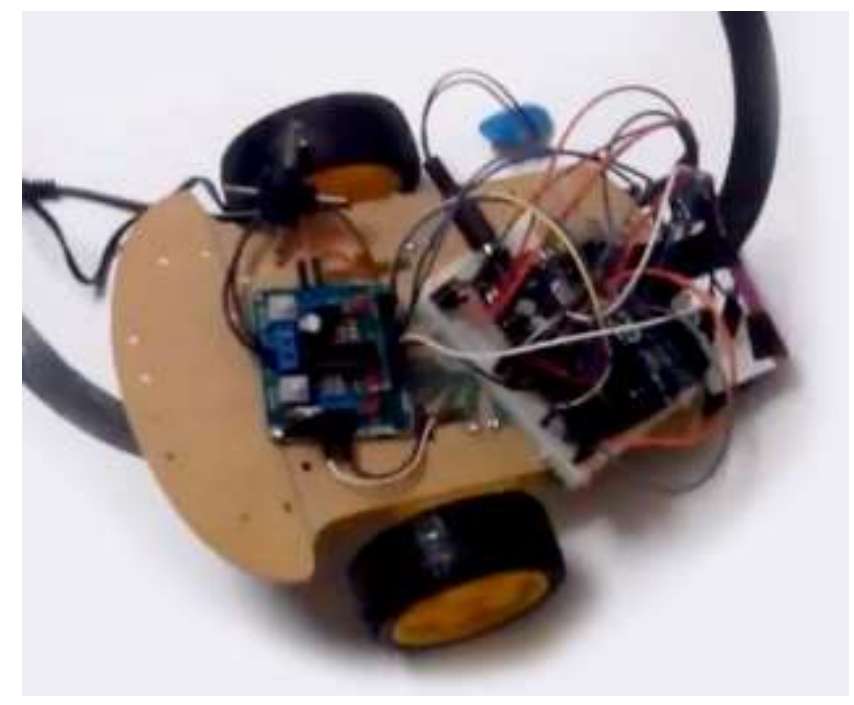

Figure 9. First Prototype 


\section{Prototype 2}

Here we started writing code to follow the line along with obstacle detection. We bought all the equipment thought it was a troublesome job because the equipment are not available everywhere. We start working on obstacle detecting basics with IR sensors. We had to change the sensor because it wasn't giving us the correct values. We changed few of the components. On the $2^{\text {nd }}$ prototype we have designed the main board. We had maintained the total heights and changed the IR sensor array height for the better implementing of IR sensor. With the $2^{\text {nd }}$ prototype it will be much easier to move and can able to detect obstacles pretty well. With $2^{\text {nd }}$ prototype our purpose was to make the robot lighter, smaller and more responsive to the main objectives.

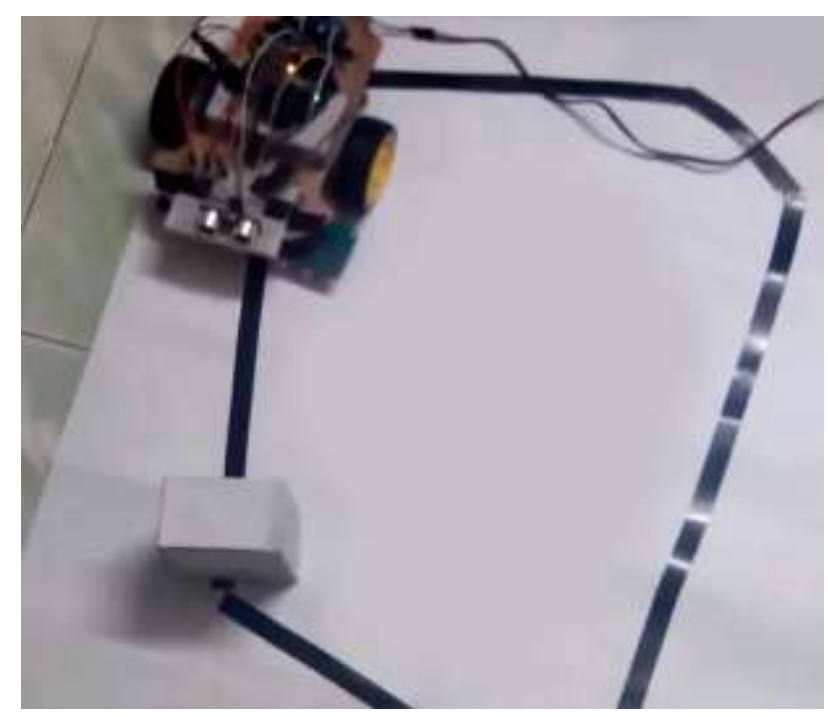

Figure 10. Second Prototype

\section{Final Prototype}

We had implemented everything on the $2^{\text {nd }}$ prototype including tested and debugged the codes. We tested all the wheels wires running through the path and developed intersection detecting algorithms. We changed the mechanical structure a bit for better accommodation. We used different surface for testing our project for the perfections and better performance. Our main problem was to run the line follower with better performances. Later it worked after changing the height. For the wireless communication we connected the nRF24L01 RF board with the Arduino Mega.
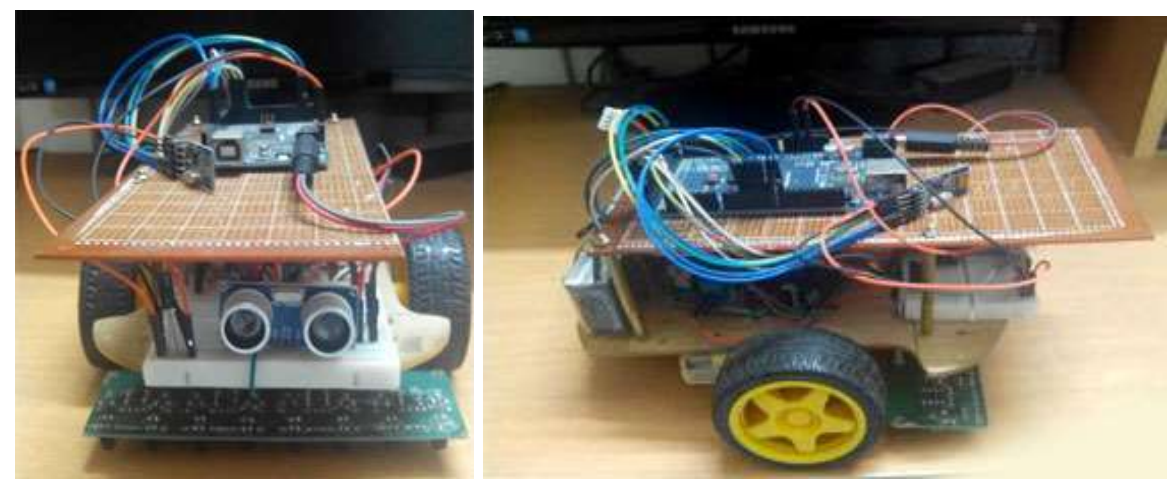

Figure 11. Final Prototype 
After testing the robot we noted down the desired readings, checked the performances and update the few lines of codes due to the environment changes.

All the description in the paper we tested all the wheels wires running through the path and developed intersection detecting algorithm. We changed the mechanical structure a bit. We connected the nRF24L01 RF board. After testing the robot we noted down the desired readings.

\section{Prototype of Base Station}

For base station we designed a very simple setup. An Arduino Uno is connected with a computer using USB cable. An nRF24L01 is connected with the Arduino Uno. At first we used the Arduino Uno's 3.3V power pin to power the nRF24L01. But it was not working well because of the shaky power supply from the Arduino Uno. Then we connected a $10 \mu \mathrm{F}$ capacitor and it solved the problem. We have used a mini breadboard to connect the capacitor with the nRF24L01. In open area the communication distance of the nRF24L01 is almost no loss within 55 meters.

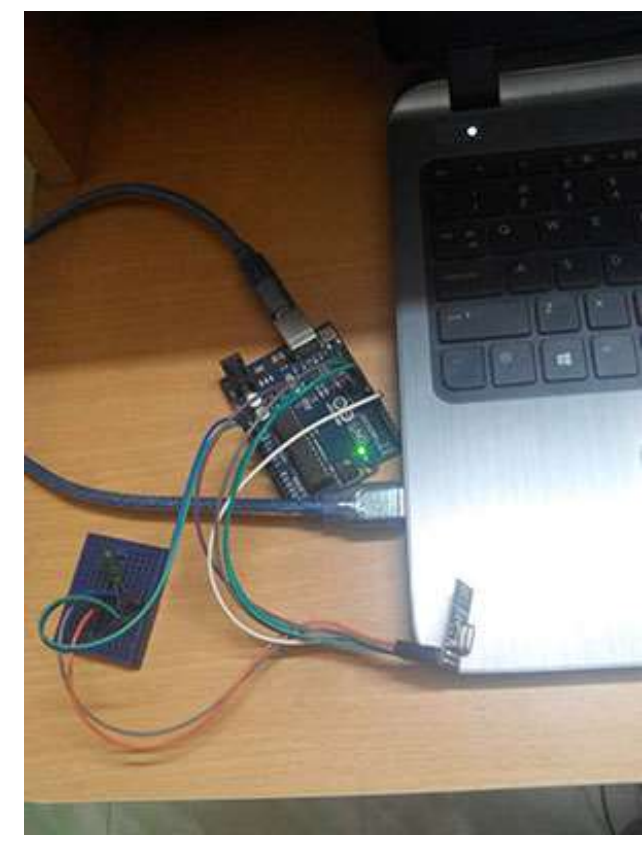

Figure 12. Base Station

\section{Outputs \& Results}

At first we have taken the individual test for each sensor, where all the sensors showed the perfect results. At the beginning the IR sensor took the reading of the black and white line analog readings, the sonar sensor gave the reading of the distances of the obstacles. We have tested the path finder robot by changing the IR sensor array distance with the surface. Firstly we kept the IR sensor array $3 \mathrm{~cm}$ above the surface. Later we kept the IR sensor array $1.5 \mathrm{~cm}$ above the surface. At the end we kept the IR sensor array $1 \mathrm{~cm}$ above the surface. The testing for the three different distances between the IR sensor array and the ground gave different outcomes. The sonar sensor is designed when any obstacles object detects around $2-20 \mathrm{~cm}$. The nRF24L01 also worked pretty well. 


\section{Average Filter Algorithm}

It receives the sonar measurements and returns the average of the measurements.

Table 2. Average Filter Algorithm

\begin{tabular}{|l|}
\hline AvgFilter \\
\hline Function avg = AvgFilter(x) \\
persistent prevAvg $\mathrm{k}$ \\
persistent firstRun \\
if isempty(firstRun) \\
$\mathrm{k}=1 ;$ \\
prevAvg $=0$ \\
firstRun $=1 ;$ \\
end \\
alpha $=(\mathrm{k}-1) / \mathrm{k}$ \\
avg $=$ alpha*prevAvg $+(1-\text { alpha })^{*} \mathrm{x}$ \\
prevAvg $=$ avg; \\
$\mathrm{k}=\mathrm{k}+1 ;$
\end{tabular}

\section{GetMaxSonar Algorithm}

It reads the sonar from a file that has a real data taken from the front sonar of the Bot.

\section{Table 3. Get Max Sonar Algorithm}

\begin{tabular}{|l|}
\hline GetMaxSonar \\
\hline function $\mathrm{h}=$ GetMaxSonar1( $)$ \\
\%persistent son $1 \%$ \\
SonartAlt.mat \\
persistent $\mathrm{k}$ firstRun \\
if isempty(firstRun) \\
load Son1 \\
$\mathrm{k}=1 ;$ \\
firstRun $=1 ;$ \\
end \\
$\mathrm{h}=\operatorname{son} 1(\mathrm{k})$ \\
$\mathrm{k}=\mathrm{k}+1 ;$ \\
\hline
\end{tabular}

\section{Result of Kalman Filer}

As shown in GetMaxSonar and AvgFilter codes, the function simply adds random values to the initial value and sends them as simulated measurements with noise. The main code is shown in Figure 4.12. This code gets the measurements from Sonar1 and Sonar2 and passes them to Kalman Filter algorithm. After getting the estimation, it will show the results. Figure 4.13 shows the result of Kalman Filer that is used in this example. 
Table 4. Result of Kalman Filter
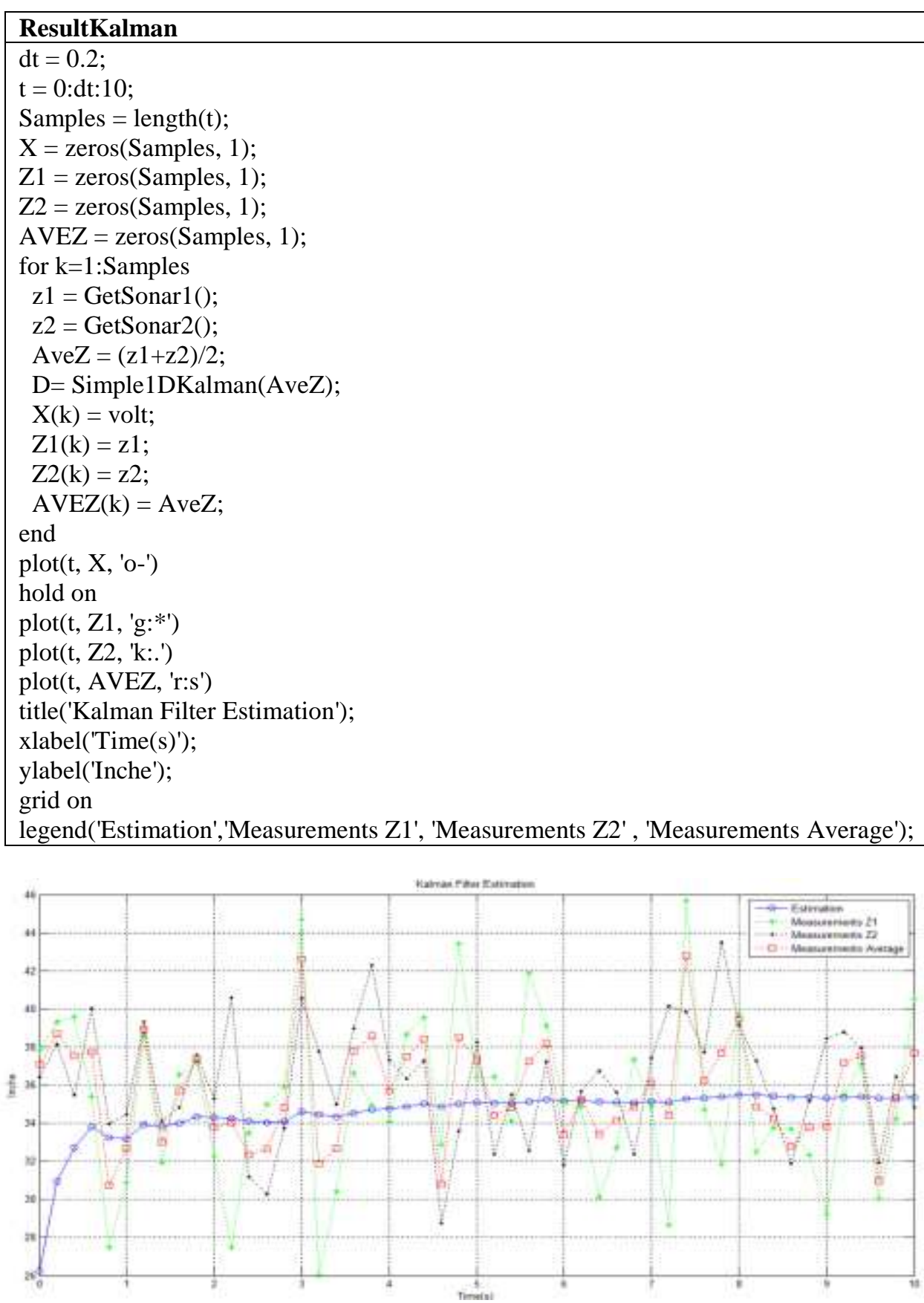

Figure 13. Kalman Filter Output 
Table 5. Accuracy Test

\begin{tabular}{|c|c|c|c|c|}
\hline \multirow{2}{*}{$\begin{array}{c}\text { No of } \\
\text { Sensors }\end{array}$} & \multirow{2}{*}{ Sensors } & \multicolumn{3}{|c|}{ Accuracy Checking } \\
\hline & & Test 1 & Test 2 & Test 3 \\
\hline 1 & $\begin{array}{l}\text { IR sensor } \\
\text { array }\end{array}$ & $\begin{array}{c}65 \% \\
\text { (didn't follow } \\
\text { the line) }\end{array}$ & $\begin{array}{c}70 \% \\
\text { (detected the } \\
\text { black line well) }\end{array}$ & $\begin{array}{c}82 \% \\
\text { (detected the black line } \\
\text { as expected) }\end{array}$ \\
\hline 2 & $\begin{array}{l}\text { Sonar } \\
\text { sensor }\end{array}$ & $\begin{array}{c}93 \% \\
\text { (object detected } \\
\text { properly) }\end{array}$ & $\begin{array}{c}95 \% \\
\text { (object detected } \\
\text { properly) }\end{array}$ & $\begin{array}{c}95.5 \% \\
\text { (object detected } \\
\text { properly) }\end{array}$ \\
\hline 3 & nRF24L01 & $\begin{array}{c}88 \% \\
\text { (data received \& } \\
\text { sent properly) }\end{array}$ & $\begin{array}{c}94 \% \\
\text { (data received } \\
\& \text { sent } \\
\text { properly) }\end{array}$ & $\begin{array}{l}95 \% \\
\text { (data received \& sent } \\
\text { properly) }\end{array}$ \\
\hline
\end{tabular}

According to the analysis we have seen that all of our sensors and communication device giving the perfect reading. For perfecting behavior of the robot we tested on 3 different environments. The sonar sensor gave $95.5 \%$ accuracy which means it can able to detect any object that causes as obstacles to the path. IR sensors gave the $82 \%$ accuracy; followed the line on three different distances with the surface and can successfully detect the black line and intersection points. According to the analysis we have found that the nRF24L01 sometimes make trouble in communication if it face shaky power supply. It is best to use a capacitor to solve the problem.

\section{Conclusions}

In its current form robot is enough capable that it can follow any line. We must build a robot that has light weight and fast, because points are awarded based upon the distance covered and the speed of the overall robot. Therefore, we used two speed motors and high sensitivity sensors circuits. Before making this kind of project it is required to relocate the perfect resources and have best information about those parts. Though it has some limitations on physical and mechanical parts but the simple robot is more effective and profitable for people. Implementing this project based on path finder can make our life easier. It can be modified and upgraded with new features and options regarding its usability. This product is economically cheaper. It's a step creating revolution implementing robots in our household needs and needs of other places.

\section{Future Direction}

In future we can incorporate the new features in our project. These features will enhance the usability of line following robot and enhance the angle of working sector. Through image processing system we can train our robot to pick up something on its own. Therefore voice synchronization will make the machine more flexible which will help us 
to call the robot from a long distance and can able to communicate with robot via voice; it is almost like talking with your own robot. Wireless control will conquer distance and make it more efficient. In Future for recharging the battery it could be possibly to use the wireless battery charger. Moreover this system could be more useful for the large shopping mart, 5 star hotels, restaurants, airports and etc. in future we can implement this system on vacuum cleaner, autonomous waiter. Most importantly after complete the implementations for all these projects we can create and apply new learning algorithms for the autonomous car by taking command from user which can drive self.

\section{References}

[1] C. Adams, Is 'dead reckoning' short for 'deduced reckoning'?, (2002).

[2] O. Q. Mohsin, "Mobile Robot Localization Based on Kalman Filter", (2014).

[3] K. Arras and N. Tomatis, "Improving robustness and precision in mobile robot localization by using laser range nding and monocularvision, Proceedings of the Third European Workshop on Advanced Mobile Robots, Zurich, Switzerland, (1999).

[4] H. Baltzakis and P. Trahanias, "Hybrid mobile robot localization using switching state space models", Proceedings of the 2002 IEEE International Conference on Robotics and Automation. Washington D.C., USA, (2002).

[5] J. Borenstein, "Control and kinematic design of multi-degree-of-freedom robots with compliant linkage”, IEEE Transactions on Robotics and Automation, (1995).

[6] J. Borenstein, H. Everett, L. Feng, and D. Wehe, "Mobile robot positioning: Sensors and techniques", Journal of Robotic Systems, vol. 14, no. 4, (1997).

[7] J. Borenstein and L. Feng, "Measurement and correction of systematic odometry errors in mobile robots", IEEE Transactions on Robotics and Automation, vol. 12, (1996).

[8] H. Bruyninkx, "Bayesian probability", (2002).

[9] W. Burgard, D. Fox, D. Hennig, and T. Schmidt, "Estimating the absolute position of a mobile robot using position probability grids", Proceedings of the Fourteenth National Conference on Artificial Intelligence, (1996).

[10] A. R. Cassandra, L. P. Kaelbling and J. A. Kurien, "Acting under uncertainty: Discrete bayesian models for mobile robot navigation", Proceedings of IEEE/RSJ International Conference on Intelligent Robots and Systems, (1996).

[11] D. Floreano and J. Urzelai, "Evolutionary robots with on-line self-organization and behavioral fitness", Neural Networks, vol. 13, no. 4-5, (2000), pp. 431-443.

[12] P. Funes, B. Orme and E. Bonabeau, "Evolving emergent group behaviors for simple humans agents", In Banzhaf, W., Christaller, T., Dittrich, P., Kim, J. T., and Ziegler, J., editors, Advances in Artificial Life. Proceedings of the 7th European Conference on Artificial Life (ECAL 2003), volume 2801 of Lecture Notes in Artificial Intelligence, Berlin: Springer Verlag, (2013), pp. 76-89.

[13] S. Roumeliotis and G. Bekey, "Bayesian estimation and kalman filtering: A unied framework for mobile robot localization", Proceedings of IEEE International Conference on Robotics and Automation. SanFrancisco, California, (2000).

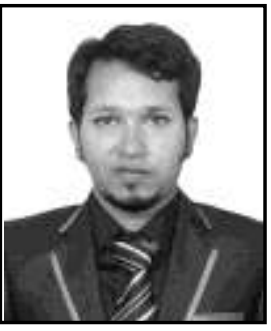

\section{Authors}

Tanvir Ahmed, is a popular web application developer in Bangladesh. He takes B.Sc. Engg. in CSE from Bangladesh University of Business \& Technology (BUBT), one of the topmost private university of Bangladesh. He is a talented computer scientist. Now he worked with a software company named RAMS ITECH as a senior software engineer. His research interests are Internet Security, Data mining, Web database, Artificial Intelligence \& Image processing. 

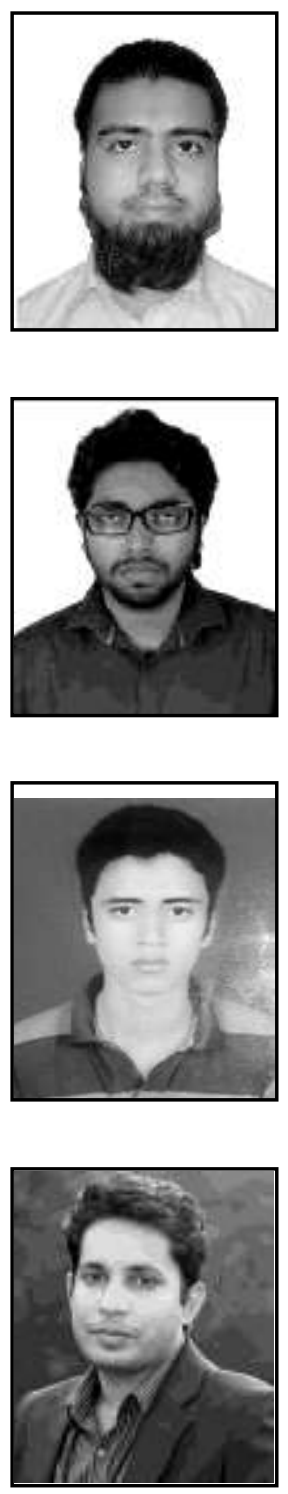

Khandakar Mahbubul Islam, is a final year student of B.Sc. in CSE from Bangladesh University of Business \& Technology (BUBT). He is a web developer and work as a freelance web app developer. Now he is completing his final year project $\&$ thesis. His research interests are Robotics, Data mining, Web database, Artificial Intelligence \& Image processing.

Faiz Ahamed, is a final year student of B.Sc. in CSE from Bangladesh University of Business \& Technology (BUBT), one of the topmost private university of Bangladesh. He is a talented software engineer of a software company named "iNiLABS". Now he is completing his final year project \& thesis. His research interests are Robotics, Data mining, Web database, Artificial Intelligence, Cloud Computing \& Security.

Rakib Hossain, is a final year student of B.Sc. in CSE from Bangladesh University of Business \& Technology (BUBT), one of the topmost private university of Bangladesh. Now he is completing his final year project $\&$ thesis. His research interests are Robotics, Data mining, Web database, Artificial Intelligence, Cloud Computing $\&$ Security.

Mahbubur Rahman, has been lecturing in CSE since mid of 2011, he received his B. Sc. Engg. in CSE from Patuakhali Science and Technology University in 2011 and M.Sc. Engg. in CSE at Bangladesh University of Engineering and Technology(BUET), Bangladesh. He is now serving one of the top most private Universities in Bangladesh named Bangladesh University of Business and Technology (BUBT). His research interests are Digital Forensics, Secure and Trustworthy Computing, Data mining, Graph theory, Neural Network. 